\title{
Monitoring of aphid flight activities in seed potato crops in Serbia
}

\author{
Andja Vučetić', Tanja Vukov², Ivana Jovičić', Olivera Petrović-Obradovićl \\ I University of Belgrade, Faculty of Agriculture, Nemanjina 6, Zemun, Belgrade, Serbia 2 University of Bel- \\ grade, Institute for Biological Research "Siniša Stankovic", Belgrade, Serbia \\ Corresponding author: Andja Vučetić (avucetic@agrif.bg.ac.rs)
}

Academic editor: E. Tasheva | Received 14 November 2012 | Accepted 31 May 2013 | Published 30 June 2013

Citation: Vučetić A, Vukov T, Jovičić I, Petrović-Obradović O (2013) Monitoring of aphid flight activities in seed potato crops in Serbia. In: Popov A, Grozeva S, Simov N, Tasheva E (Eds) Advances in Hemipterology. ZooKeys 319: $333-346$. doi: $10.3897 /$ zookeys.319.4315

\begin{abstract}
Aphid flight activities in seed potato fields have been studied by the yellow water traps. It is a good method for monitoring aphids as vectors of viruses, but this study also showed it is a suitable method for insectdiversity research. During the four-year studies, over 11.500 specimens were collected and a total of 107 different taxa of aphids were identified. The most abundant species were polyphagous species, such as: Acyrthosiphon pisum (Haris), Aphis fabae Scopoli, Aphis gossypii Glover and Brachycaudus helichrysi (Kaltenbach). The results of the studies show that diversity of aphids in different regions of Serbia is similar regardless of the altitude and the diversity of terrain. At most sites it ranged from 2 to 3 . The highest value was recorded in Begeč, locality in northern part of Serbia, in year 2008, and it was 2.92. The maximum values of the Shannon-Weaver diversity index at all sites were recorded in the first weeks of the monitoring of aphid flight activities. Morisita-Horn similarity index shows no significant differences between sites regardless of altitudes. The sites are grouped by year, not by similarity of relief. In spite of these results, the Chi-square analysis showed highly significant difference in vector frequencies among seasons and sites with more pronounced differences for PVY. As a consequence of differences in vector frequencies, the vector pressure index in some regions was different also. The number of vectors and vector pressure index vary depending on the altitude of localities. At localities at altitudes under 1000 m, they were high. The highest index was at Kotraža, locality in central part of Serbia, in 2007, when PVY index exceeded the value of 180, while for PLRV it was 60. At high altitudes on mountain Golija, above $1100 \mathrm{~m}$, the number of aphids was low, as well as the vector pressure index which indicates that these regions are suitable for producing virus-free seed potato.
\end{abstract}

\section{Keywords}

Aphids, potato, Shannon-Weaver index diversity, Morisita-Horn similarity index, vectors of viruses

Copyright Andja Vučetić et al. This is an open access article distributed under the terms of the Creative Commons Attribution License 3.0 (CC-BY), which permits unrestricted use, distribution, and reproduction in any medium, provided the original author and source are credited. 


\section{Introduction}

Aphids (Aphididae, Hemiptera) are the most efficient vectors of plant pathogenic viruses therefore they cause serious problems in potato growing. Production of healthy seed potatoes is possible in conditions of reduced number of aphids and their ability to come into contact with the plant and transfer the virus (Robert and Bourdin 2001). Two most important potato viruses are Potato Virus Y (PVY) and Potato Leafroll Virus (PLRV). Seed quality depends directly on the infection level (Salazar 1996).

After infection of leaves, the virus is translocated into the tubers. In some countries, earlier sowing and haulm destruction is carried out at critical period of virus infection (Van Harten 1983). It is a good way to stop virus transmission from leaves to tubers. In Serbia the maximum aphid flight activities and at the same time the maximum vector activities occur end of May-early June (Petrović-Obradović 2003). In that period, potato is at early stages of growth and desiccation is not possible. That can completely interrupt plant's vegetation and the yield would be lost. Because of similar vegetation complexity, relief, climate and aphid fauna, situation is similar in neighboring countries in southeastern Europe. In this region, it is necessary to find some other way for the production of healthy seed potato. One possibility is to find localities with a lower number of aphids and inoculum sources. The success of agricultural production depends on the biodiversity of an area, the number of present organisms, which may have a positive or a negative impact (Dueli 1997, Laznik et al. 2010). Diversity of aphids is just a segment of biodiversity of an area, but significant in the aspect of ecology and crop production, in this case production of seed potatoes.

The aim of these studies was to determine the biodiversity of aphids and similarity in aphid composition between different regions of Serbia. Also, the aim of these studies was to determine differences in vectors frequency among different sites in Serbia and to calculate the pressure of vectors for the two most important potato viruses (Potato Virus Y - PVY and Potato Leafroll Virus - PLRV), and thus determine which areas are suitable for the cultivation of healthy seed potatoes.

\section{Material and methods}

Aphid flight activity was studied in different areas of Serbia in twenty sites for four years (2007-2010). These 20 sites belong to the three major potato growing areas in Serbia. The first area is in northern part of Serbia under the altitudes of $80 \mathrm{~m}$ (localities: Begeč, Stanišić, Kupusina). The second one is in central part at altitudes of $400-$ 900 m (localities: Kotraža, Zablaće, Prijevor, Glumač), and the third one is in southern part at altitudes above $1100 \mathrm{~m}$ (localities on mountain Golija) (Fig. 1). Monitoring of aphid flight activity was conducted by using yellow water traps. Yellow water traps were placed in potato crops (4traps/1ha) immediately after the emergence of potato. Traps have been raised gradually to be visible for aphids during the growth of the crop. Samples were taken once per week until drying of the above-ground mass. Aphids were 


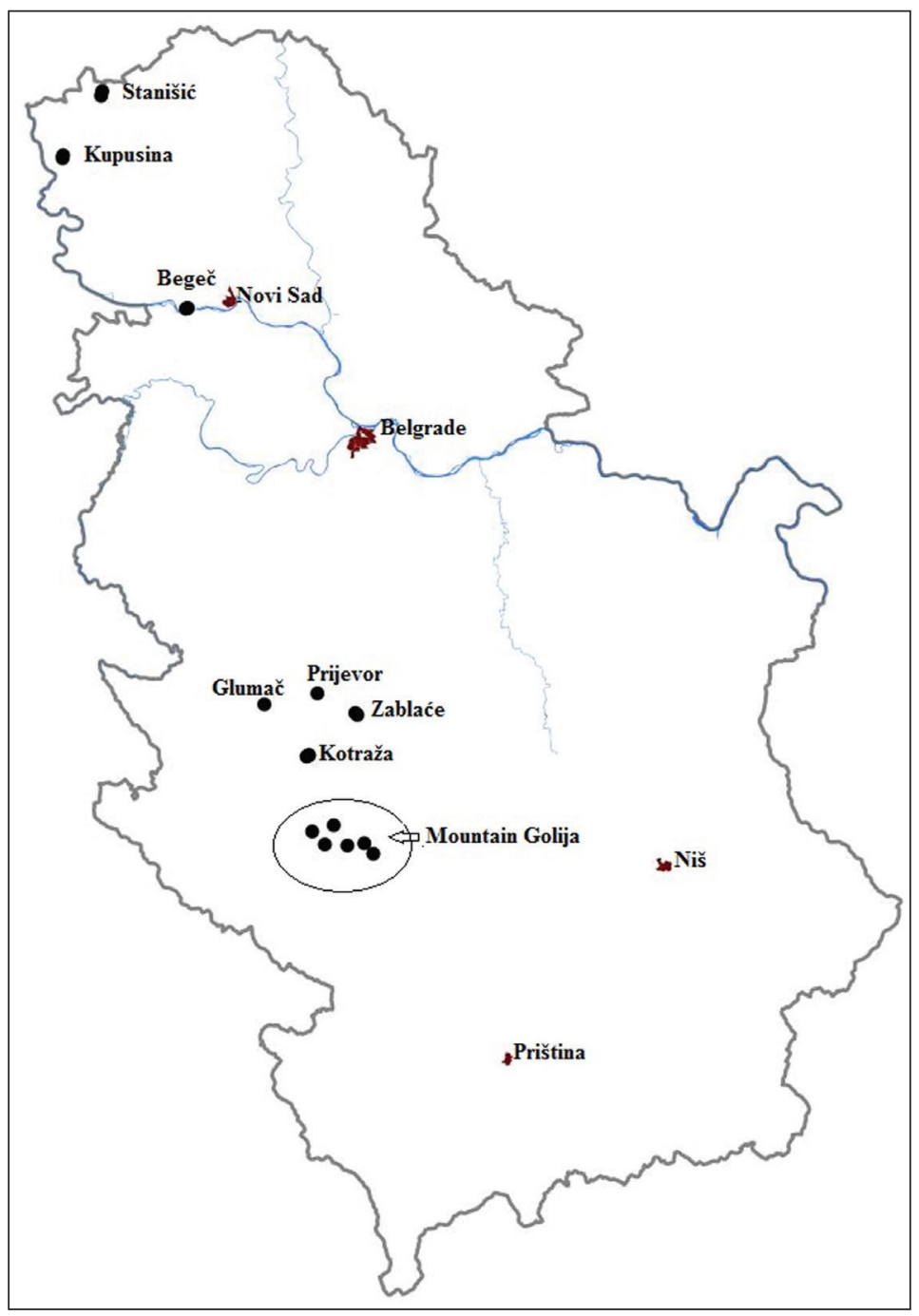

Figure I. Map of Serbia with monitored aphid flight activities sites. Coordinates of localities: Begeč

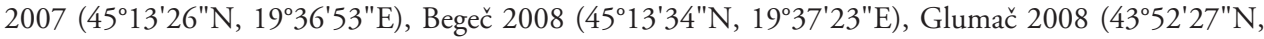

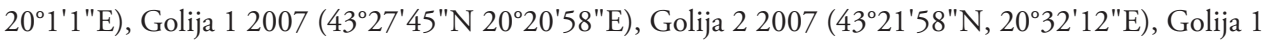

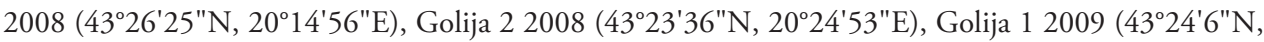
20²9'38"E), Golija 22009 (4323'48"N, 20¹8'31"E), Kotraža 2007 (4342'7"N, $20^{\circ} 13^{\prime} 49^{\prime \prime E}$ ), Kotraža

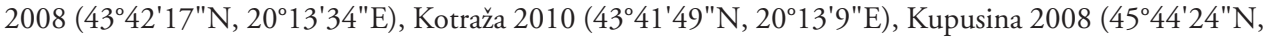

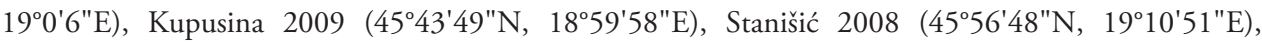

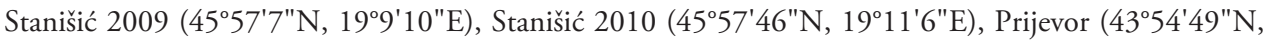
$20^{\circ} 16^{\prime 2} 2^{\prime E}$ ), Zablaće 22009 (4350'51"N, 20²6'51"E), Zablaće 12010 (4350'27"N, 20²7'25"E).

identified using a stereoscopic microscope (Bio-optica, Italy, Type: 1000) and keys for identification of alatae aphids (Taylor 1984, Jacky and Bouchery 1988, Remaudiere and Seco Fernandez 1990). 
Shannon-Weaver index was used for the analysis of biodiversity (Krebs 1989). A diversity index is a quantitative measure that reflects how many different species there are in a dataset, and simultaneously takes into account how evenly individuals are distributed among those types. It is always ranging between 0 (indicating low community complexity) and 4 (indicating high community complexity). Morisita-Horn similarity index was used to calculate the similarities in aphid composition among the sites (Magurran 2004). This index takes into account composition and richness of fauna and successfully compares samples of different size. The maximum value of this index is 1 . The cluster analysis on the basis of Morisita-Horn index after the UPGA method was conducted. Comparisons of number of aphids that are vectors for PVY and PLRV viruses among localities were performed with Chi-square analysis. Similarity Percentage analysis (SIMPER) (Clarke 1993) was applied for determination of the contribution of each aphid species, which are identified as virus vectors, to the similarity (the dissimilarity) between all localities and years of collections. The Bray-Curtis measure was calculated after logarithmic transformation of the data. The risk of infection of potato by viruses was shown as a cumulative index vector pressure which was calculated using the Relative Transmission Efficiency value of aphids, known vectors of potato viruses (http://aphmon.csl.gov.uk/info.cfm).

\section{Results}

During the four-year studies, over 11.500 specimens were collected and a total of 107 different taxa of aphids were identified. Seventy five different taxa were identified to the species level, thirty two to genus level. Thirty six heteroecious species and thirty nine monoecious species were identified (Table 1). According to the data PetrovićObradović (2003), 60 aphid species, which is about 17\% of identified species in Serbia, are heteroecious. During these researches, almost $50 \%$ of identified species were heteroecious, which are commonly polyphagous species and some of them are the most important vectors of potato viruses. Two invasive species were found during this research, Trichosiphonaphis polygonifoliae (Shinji), which had been recently discovered on its host plant (Petrović-Obradović et al. 2010) and Macrosiphum albifrons Essig which had been found for the first time in Serbia during this research.

Results from 20 different localities were used for the analysis of biodiversity using Shannon-Weaver index. The maximum values of the Shannon-Weaver index diversity at all sites were recorded in the first weeks of the monitoring of aphid flight activities (Fig. 2).

The results of studies show that diversity of aphids in different regions of Serbia is similar regardless of the altitude and the diversity of terrain. At most sites, it ranged from 2 to 3. The highest value was recorded in Begeč in 2008, where it was 2.92. The lowest values were on the mountain Golija in 2008, where on the locality at the lower altitudes was 0.69 and at the higher altitudes was 1.098. In that year, in two localities on this mountain, a total of 5 aphid specimens were caught (Fig. 3). 
Table I. Identified aphid taxa ( ${ }^{\mathrm{PVY}}$ vectors of PVY, ${ }^{\text {PLRV }}$ vectors of PLRV).

\begin{tabular}{|c|c|c|}
\hline Monoecious species & Heteroecious species & Genus \\
\hline Acyrthosiphon cyparissiae (Koch) & Anoecia corni (F.) & Acyrthosiphon spp. \\
\hline Acyrthosiphon malvae (Mosley) & Aphis fabae Scopoli PVY /PLRV & Amphorophora spp. \\
\hline Acyrthosiphon pisum (Haris) ${ }^{\mathrm{PVY}}$ & Aphis gossipii Glover ${ }^{\text {PLRV }}$ & Anoecia spp. \\
\hline Amphorophora rubi (Kaltenbach) & Aphis nasturtii Kaltenbach & Aphis spp. \\
\hline Aphis craccivora Koch & Aphis spiraecola Patch & Brachycaudus spp. \\
\hline Aphis idaei van der Goot & Aphis sambuci $\mathrm{L}$ & Capitophorus spp. \\
\hline Aphis pomi De Geer & Aulacorthum solani (Kaltenbach) ${ }^{\text {PVY /PLRV }}$ & Cavariella spp. \\
\hline Atheroides serrulatus Haliday & Brachycaudus cardui (L.) & Chaitophorus spp. \\
\hline Brevycorinae brassicae (L.) & Brachycaudus helichrysi (Kaltenbach) ${ }^{\mathrm{PVY}}$ & Cinara spp. \\
\hline Callipterinela calliptera (Hartig) & Capitophorus eleagni (del Guercio) & Dysaphis spp. \\
\hline Callipterinela tuberculata (von Heyden) & Cavariella theobaldi (Gillete and Bragg) & Eriosoma spp. \\
\hline $\begin{array}{l}\text { Chaitophorus populialbe } \\
\text { (Boyer de Fonscolombe) }\end{array}$ & Cryptomyzus galeopsidis (Kaltenbach) & Euceraphis spp. \\
\hline Cinara tujafilina (del Guercio) & Cryptomyzus ribis (L.) & Forda spp. \\
\hline Ctenocallis setosus (Kaltenbach) & Dysaphis plantaginea (Passerini) & Hyadaphis spp. \\
\hline Drepanosiphum aceris Koch & Eriosoma ulmi (L.) & Hyperomyzus spp. \\
\hline Eucalipterus tiliae (L.) & Forda marginata Koch & Macrosiphoniella spp. \\
\hline Euceraphis betulae (Koch) & Hyadaphis foeniculi (Passerini) & Microlophium spp. \\
\hline Eriosoma lanigerum (Hausmann) & Hyalopterus pruni complex & Myzocallis spp. \\
\hline Hyadaphis polonica Szelegiewicz & Hyperomyzus lactuce (L.) & Myzus spp. \\
\hline Lachnus roborus $(\mathrm{L})$. & Hyperomyzus pallidus Hille Ris Lambers & Ovatus spp. \\
\hline Lipaphis erysimi (Kaltenbach) & Hyperomyzus picridis (Börner and Blunck) & Pemphigus spp. \\
\hline Macrosiphum albifrons Essig & Macrosiphum euphorbiae (Thomas) ${ }^{\text {PVY /PLRV }}$ & Periphyllus spp. \\
\hline Macrosiphum funestrum (Macchiati) & Macrosiphum rosae (L.) & Protaphis spp. \\
\hline Megoura viciae Buckton & Metopolophium dirhodum (Walker) ${ }^{\mathrm{PVY}}$ & Protrama spp. \\
\hline Megourella purpurea Hille Ris Lambers & Myzus cerasi (Fabricus) & Rhopalosiphum spp. \\
\hline Myzocallis castanicola Baker & Myzus persicae (Sulzer) ${ }^{\text {PVY /PLRV }}$ & Semiaphis spp. \\
\hline $\begin{array}{l}\text { Myzocallis occidentalis Remaudie et } \\
\text { Nieto Nafria }\end{array}$ & Nasonovia ribis-nigri (Mosley) & Sipha spp. \\
\hline Myzodium modestum (Hottes) & Phorodon humuli (Schrank) ${ }^{\text {PLRV }}$ & Subsaltusaphis spp. \\
\hline Myzus ligustri (Mosley) & Rhopalomyzus poae (Gill) & Tetraneura spp. \\
\hline Ovatus inulae (Walker) & Rhopalosiphoninus staphylleae (Koch) ${ }^{\text {PLRV }}$ & Therioaphis spp. \\
\hline Phyllaphis fagi (L.) & Rhopalosiphum maidis (Fitch) & Tuberculatus spp. \\
\hline Pterocallis alni (de Geer) & Rhopalosiphum nimfaeae (L.) & Uroleucon spp. \\
\hline Schizaphis graminum (Rondani) & Rhopalosiphum padi (L.) ${ }^{\mathrm{PVY}}$ & \\
\hline Sipha elegans del Guercio & Sitobion fragariae (Walker) & \\
\hline Sipha maydis Passerini & Smynthurodes betae Westwood & \\
\hline Sitobion avenae (Fabricius) & Trichosiphonaphis polygonifoliae (Shinji) & \\
\hline \multicolumn{3}{|l|}{ Therioaphis trifolii (Monell) } \\
\hline \multicolumn{3}{|l|}{ Tinocallis platani (Kaltenbach) } \\
\hline $\begin{array}{l}\text { Wahlgreniella ossiannilssoni Hille } \\
\text { Ris Lambers }\end{array}$ & & \\
\hline
\end{tabular}




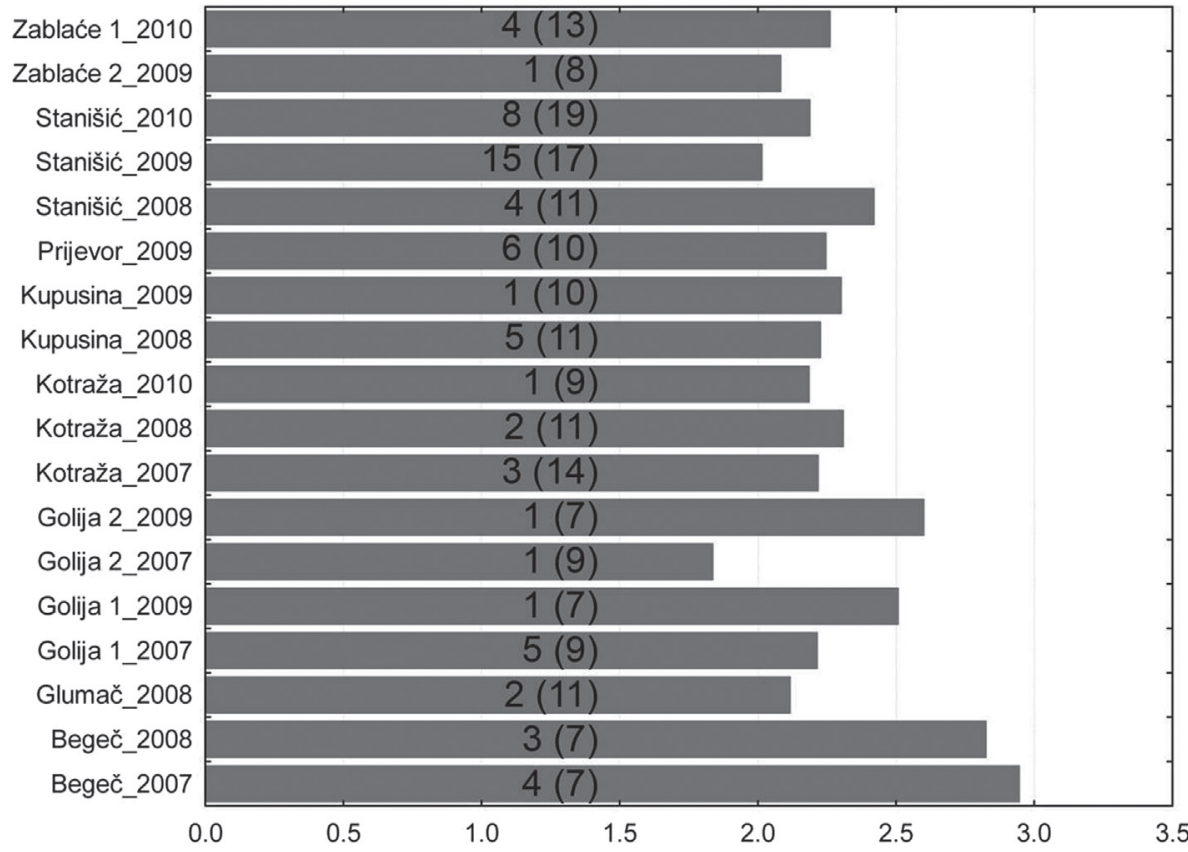

Figure 2. Maximum of Shannon-Weaver index per locality (number in brackets - number of weeks of monitoring aphid flight activities, number without brackets - week with maximum value of ShannonWeaver index).

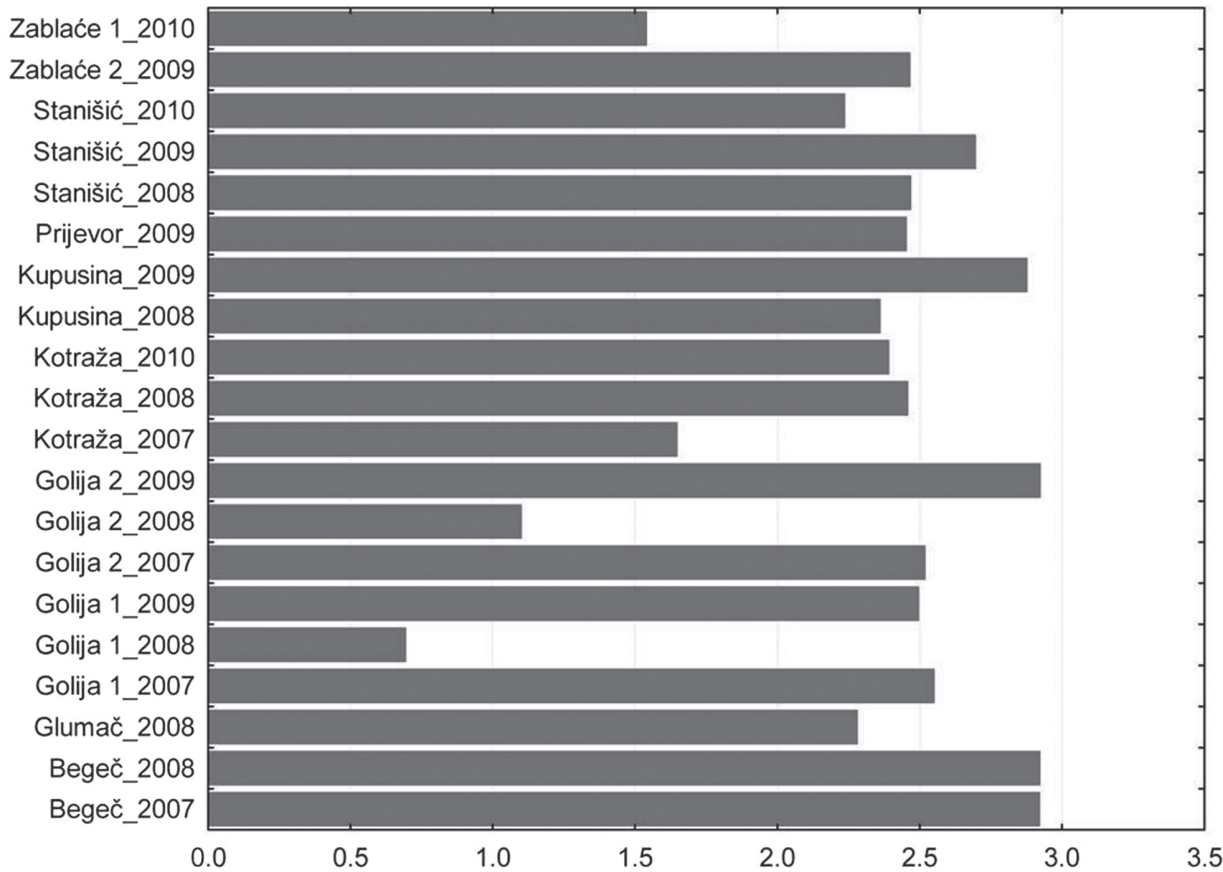

Figure 3. Total Shannon-Weaver index diversity per locality. 


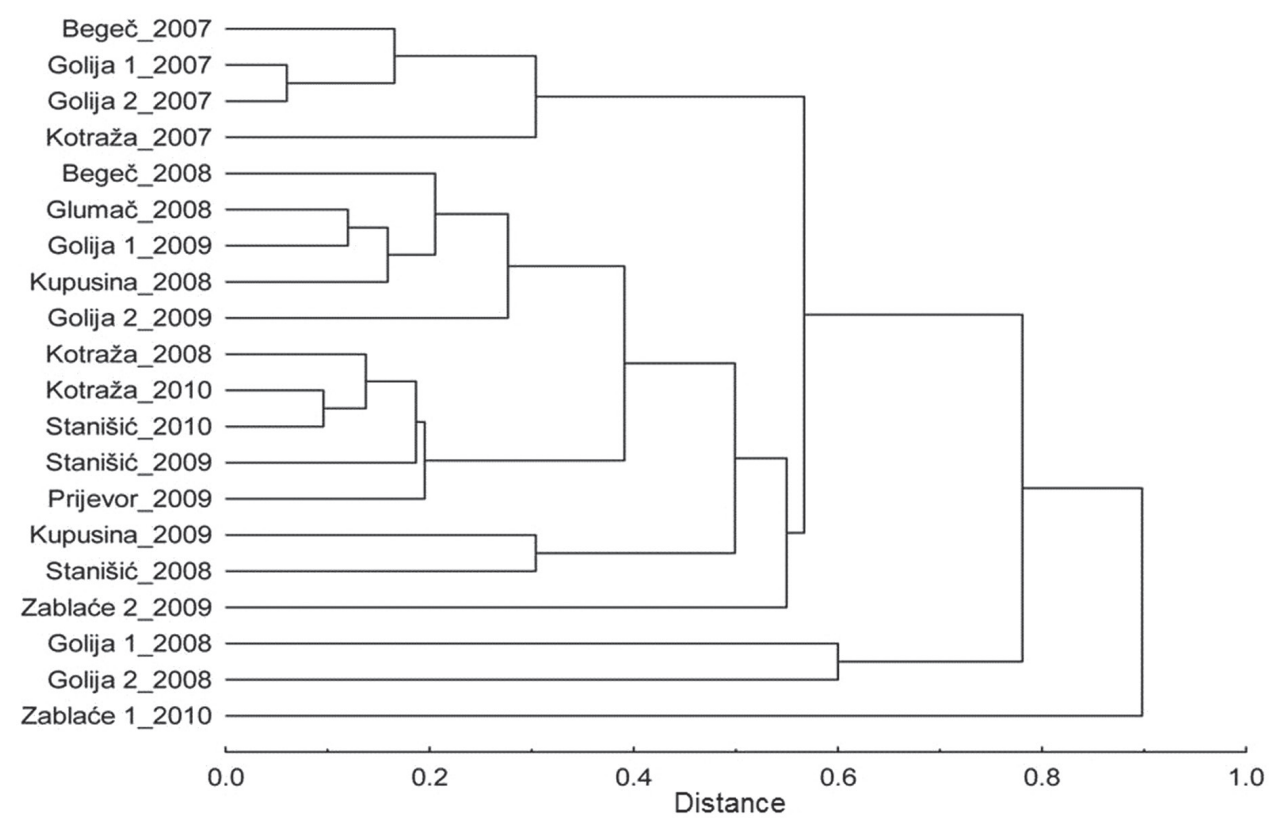

Figure 4. Dendrogram shows similarity between the sites, constructed on the basis of Morisita-Horn similarity index.

Also, Morisita-Horn similarity index shows no significant differences in aphid composition between sites regardless of altitudes. The cluster analysis on the basis of this index was carried out (Fig. 4). The sites are grouped by year, not by similarity of relief. Sites on the mountain Golija were clearly separated from the rest because of the low number of aphids in the year 2008. In the locality Zablaće, five aphid species were caught which were not recorded previously in other localities during these studies. These species are: Chaitophorus populialbae (Boyer de Fonscolombe), Myzocallis castanicola Baker, Myzocallis occidentalis Remaudie et Nieto Nafria, Protrama spp. and Sminthuroides betae Westwood.

Important potato virus vectors such as: Acyrthosiphon pisum (Haris), Aphis fabae Scopoli, Aphis gossypii Glover, Aulacorthum solani (Kaltenbach), Brachycaudus helichrysi (Kaltenbach), Macrosiphum euphorbiae (Thomas), Metapolophium dirhodum (Walker), Myzus persicae (Sulzer) and Rhopalosiphum padi (L.) were found in almost all localities, but in different numbers. The most important vector of potato viruses M. persicae, was found in all localities, but in high number just in localities Begeč and Kotraža in 2007.

The Chi-square analysis showed highly significant difference in vector frequencies among seasons and sites, with more pronounced differences for PVY (Table 2).

Similarity Percentage analysis shown that similarities in presence of PVY vectors between localities in 2007 year was almost 60\%. In that year the most common species was Br. helichrysi, which was dominant species in all localities, but in localities 


\begin{tabular}{|c|c|c|c|c|c|c|c|c|c|c|c|c|c|c|}
\hline 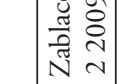 & $\stackrel{*}{*}$ & 芰 & 芰 & 娄 & $\stackrel{*}{*}$ & * & $\stackrel{*}{*}$ & $\underset{*}{*}$ & $\stackrel{*}{*}$ & * & 娄 & 芰 & 娄 & * \\
\hline 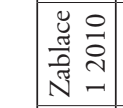 & $\underset{*}{*}$ & $\stackrel{*}{*}$ & $\stackrel{*}{*}$ & $\mathscr{\exists}$ & $\stackrel{*}{*}$ & $\tilde{z}$ & 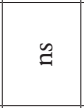 & $\stackrel{*}{*}$ & $\stackrel{*}{*}$ & $\stackrel{*}{*}$ & $\cong$ & * & 娄 & 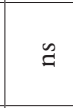 \\
\hline 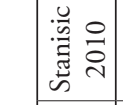 & $\stackrel{*}{*}$ & $\stackrel{*}{*}$ & $\stackrel{*}{*}$ & 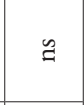 & $\stackrel{*}{*}$ & $\check{\beth}$ & $\tilde{a}$ & $\stackrel{*}{*}$ & * & 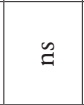 & $\stackrel{*}{*}$ & $\cong$ & $\cong$ & * \\
\hline 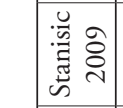 & 娄 & $\stackrel{*}{*}$ & $\stackrel{*}{*}$ & * & $\stackrel{*}{*}$ & 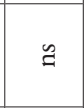 & * & 娄 & $\stackrel{*}{*}$ & $\cong$ & $\stackrel{*}{*}$ & $\mathscr{a}$ & $\cong$ & $\mathscr{Z}$ \\
\hline 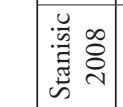 & $\stackrel{*}{*}$ & $\stackrel{*}{*}$ & $\underset{*}{*}$ & $\tilde{a}$ & $\stackrel{*}{*}$ & 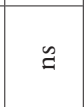 & $\cong$ & $\underset{*}{*}$ & $\stackrel{*}{*}$ & * & $\stackrel{*}{*}$ & $\check{a}$ & $\stackrel{*}{*}$ & \\
\hline 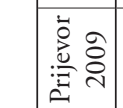 & $\stackrel{*}{*}$ & $\stackrel{*}{*}$ & $\underset{*}{*}$ & * & $\stackrel{*}{*}$ & $\cong$ & * & $\underset{*}{*}$ & * & 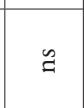 & 娄 & * & & $\stackrel{*}{*}$ \\
\hline 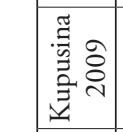 & $\begin{array}{l}* \\
* \\
*\end{array}$ & $\stackrel{*}{*}$ & $\stackrel{*}{*}$ & $\cong$ & $\underset{*}{*}$ & 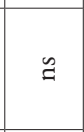 & $\mathscr{z}$ & $\stackrel{*}{*}$ & $\stackrel{*}{*}$ & $\stackrel{*}{*}$ & $\stackrel{*}{*}$ & & 娄 & $\stackrel{*}{*}$ \\
\hline 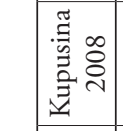 & $\stackrel{*}{*}$ & $\underset{*}{*}$ & $\underset{*}{*}$ & $\stackrel{*}{*}$ & $a$ & * & $\stackrel{*}{*}$ & 娄 & $\stackrel{*}{*}$ & $\underset{*}{*}$ & & 芰 & $\stackrel{*}{*}$ & $\stackrel{*}{*}$ \\
\hline 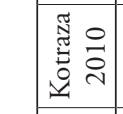 & $\underset{*}{*}$ & $\stackrel{*}{*}$ & $\stackrel{*}{*}$ & $\check{a}$ & $\stackrel{*}{*}$ & $气$ & $\check{a}$ & $\stackrel{*}{*}$ & * & & $\stackrel{*}{*}$ & $\stackrel{*}{*}$ & $\cong$ & $\stackrel{*}{*}$ \\
\hline 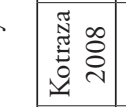 & $\stackrel{*}{*}$ & $\underset{*}{*}$ & $\stackrel{*}{*}$ & * & $\underset{*}{*}$ & * & $\underset{*}{*}$ & $\underset{*}{*}$ & & $\stackrel{*}{*}$ & * & $\stackrel{*}{*}$ & $\stackrel{*}{*}$ & $\underset{*}{*}$ \\
\hline 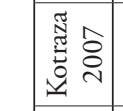 & $\underset{*}{*}$ & $\underset{*}{*}$ & $\underset{*}{*}$ & $\underset{*}{*}$ & 娄 & 芰 & 菊 & & $\stackrel{*}{*}$ & $\underset{*}{*}$ & $\underset{*}{*}$ & $\underset{*}{*}$ & $\stackrel{*}{*}$ & $\underset{*}{*}$ \\
\hline 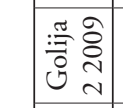 & 娄 & $\stackrel{*}{*}$ & $\stackrel{*}{*}$ & $\cong$ & 奧 & $\cong$ & & $\underset{*}{*}$ & * & $\cong$ & $\underset{*}{*}$ & $\check{g}$ & $\cong$ & $\stackrel{*}{*}$ \\
\hline 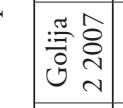 & $\stackrel{*}{*}$ & $\stackrel{*}{*}$ & $\stackrel{*}{*}$ & $\check{a}$ & $\underset{*}{*}$ & & $\underset{*}{*}$ & $\stackrel{*}{*}$ & $\begin{array}{l}\stackrel{*}{*} \\
*\end{array}$ & $\stackrel{*}{*}$ & $\stackrel{*}{*}$ & $\stackrel{*}{*}$ & $\cong$ & * \\
\hline 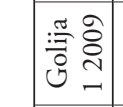 & $\underset{*}{*}$ & 娄 & $\underset{*}{*}$ & $\underset{*}{*}$ & & 娄 & $\underset{*}{*}$ & $\stackrel{*}{*}$ & $\stackrel{*}{*}$ & $\stackrel{*}{*}$ & * & $\underset{*}{*}$ & 娄 & $\stackrel{*}{*}$ \\
\hline 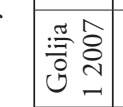 & $\stackrel{*}{*}$ & $\underset{*}{*}$ & $\underset{*}{*}$ & & 娄 & $\tilde{g}$ & $\underset{*}{*}$ & $\begin{array}{l}* \\
\stackrel{*}{*}\end{array}$ & $\stackrel{*}{*}$ & * & 娄 & 娄 & $\mathscr{a}$ & $\stackrel{*}{*}$ \\
\hline 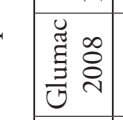 & $\stackrel{*}{*}$ & $气$ & & 荬 & 奧 & $\stackrel{*}{*}$ & 苂 & $\underset{*}{*}$ & $\stackrel{*}{*}$ & $\underset{*}{*}$ & $\underset{*}{*}$ & 娄 & 娄 & $\underset{*}{*}$ \\
\hline 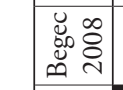 & 娄 & & * & $\stackrel{*}{*}$ & 奧 & $\stackrel{*}{*}$ & 粪 & $\stackrel{*}{*}$ & $\stackrel{*}{*}$ & $\underset{*}{*}$ & $\underset{*}{*}$ & 芰 & 娄 & $\underset{*}{*}$ \\
\hline 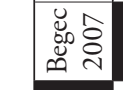 & 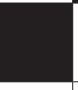 & $\underset{*}{*}$ & 娄 & $\underset{*}{*}$ & $\underset{*}{*}$ & 娄 & 粪 & $\underset{*}{*}$ & 娄 & $\stackrel{*}{*}$ & $\stackrel{*}{*}$ & $\underset{*}{*}$ & 娄 & $\stackrel{*}{*}$ \\
\hline$\underset{\nexists}{\gtrsim}$ & 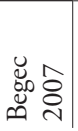 & 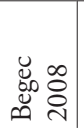 & 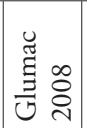 & $\left|\begin{array}{ll}\vec{\pi} & \\
: \frac{\pi}{7} & \hat{8} \\
0 & 0\end{array}\right|$ & 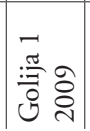 & 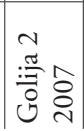 & 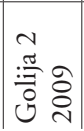 & 苂 & 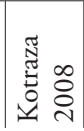 & 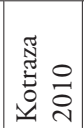 & 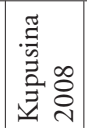 & 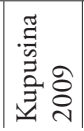 & 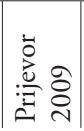 & 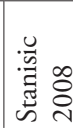 \\
\hline
\end{tabular}




\begin{tabular}{|c|c|c|c|c|c|}
\hline 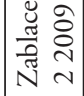 & $\stackrel{*}{*}$ & * & 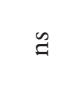 & & \\
\hline 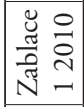 & 苂 & $\mathscr{\beth}$ & & 芰 & \\
\hline 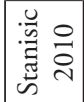 & 芰 & & $\oiiint$ & $\stackrel{*}{*}$ & \\
\hline 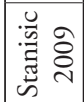 & & 㐘 & $\underset{*}{*}$ & $\underset{*}{*}$ & \\
\hline 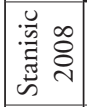 & 类 & $\stackrel{*}{*}$ & $\underset{*}{*}$ & $\stackrel{*}{*}$ & \\
\hline 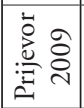 & $\underset{*}{*}$ & $\cong$ & $\cong$ & $\stackrel{*}{*} \underset{*}{*}$ & \\
\hline 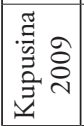 & $\stackrel{*}{*}$ & $\stackrel{*}{*}$ & * & 芰 & \\
\hline 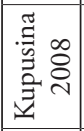 & 类 & 芰 & * & $\stackrel{*}{*}$ & \\
\hline 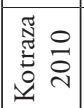 & * & $\mathscr{a}$ & $\check{z}$ & * & \\
\hline 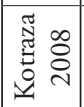 & $\stackrel{*}{*}$ & $\stackrel{*}{*}$ & * & 芰 & \\
\hline 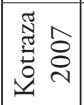 & 菊 & $\stackrel{*}{*}$ & 娄 & 芰 & \\
\hline 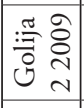 & $\stackrel{*}{*}$ & $\mathfrak{a}$ & * & $\stackrel{*}{*}$ & n \\
\hline $\begin{array}{ll}: \approx & \\
: & 0 \\
0 & 0 \\
0 & \sim \\
0\end{array}$ & $\underset{*}{*}$ & $\stackrel{*}{*}$ & 芰 & $\frac{*}{*}$ & 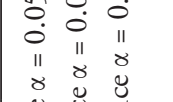 \\
\hline 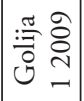 & $\underset{*}{*}$ & $\underset{*}{*}$ & $\stackrel{*}{*}$ & 芰 & 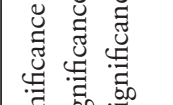 \\
\hline 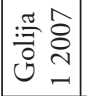 & 娄 & * & 娄 & $\stackrel{*}{*}$ & 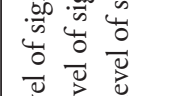 \\
\hline 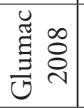 & 荬 & 荬 & $\stackrel{*}{*}$ & $\stackrel{*}{*}$ & 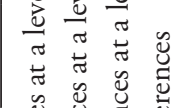 \\
\hline 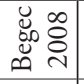 & $\stackrel{*}{*}$ & 芰 & 芰 & $\frac{*}{*}$ & 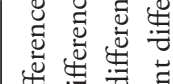 \\
\hline 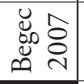 & $\stackrel{*}{*}$ & 芰 & 芰 & $\stackrel{*}{*}$ & 吾豆节 \\
\hline$\underset{a}{a}$ & : & 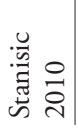 & 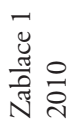 & 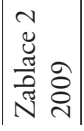 & 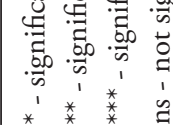 \\
\hline
\end{tabular}




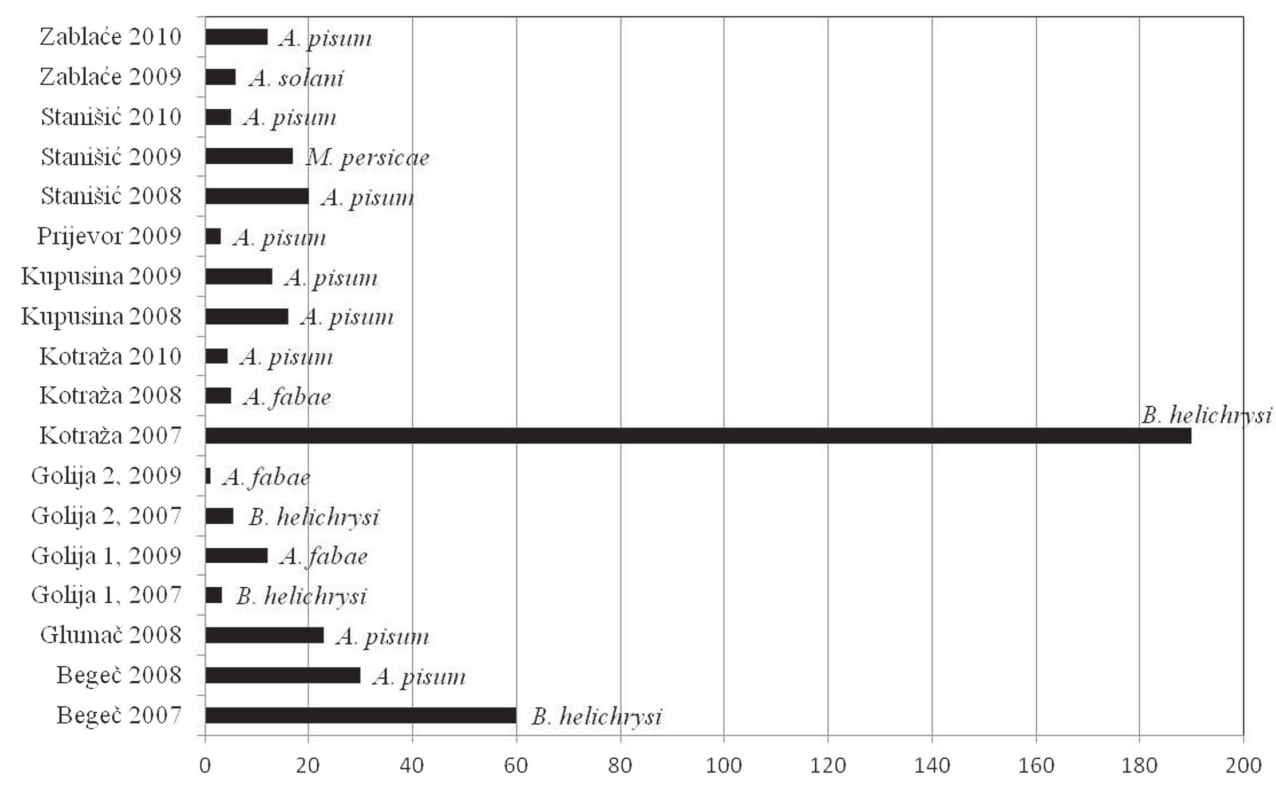

Figure 5. Vector pressure index for PVY and most important vector per locality.

Begeč and Kotraža it was found in large number. In the locality Kotraža over 1500 specimens were caught during the monitoring period. In the next years, large number of the specimens of this species has not been repeated, and because of that there was low similarity percent between localities in this year and localities in the fallowing years. In 2008 and 2010 similarity percent between localities were 70\%, but in 2009 it was $50 \%$. In all those years the most common vector species were $A$. fabae and $A$. pisum which were most responsible for high percent of similarities between localities. In all years the least number of aphids was recorded in localities in mounting Golija at altitudes above $1100 \mathrm{~m}$. There were recorded no significant differences between these localities and locality Prijevor because of low number of aphids in this locality and similar number of species $A$. fabae and $A$. solani. Similarity between localities in presence of PLRV vectors was around 70\% in 2008 and 2010. In 2007 it was $51 \%$ and in 2009 just $44 \%$. In all years the most common vector species was $A$. fabae. The best average dissimilarity was recorded between localities Begeč in 2007 and 2008 and all others localities because of constantly high number of vectors in this area. Especially high percent was recorded between this locality and localities Golija 2 in all three years, and it was 60\%.

As a consequence of differences in diversity among sites and difference in vector frequencies, the vector pressure index in some regions was different also. The lowest vector number and the lowest vector pressure were observed in Golija mountain area during the study. In localities at above $1100 \mathrm{~m}$ in Golija, vector pressure index newer exceeded 10 . The highest value was recorded in locality Kotraža in 2007 (at altitudes of $850 \mathrm{~m}$ ), when pressure of vectors for PVY exceeded 180, and for PLRV exceeded 60. In the following years, 


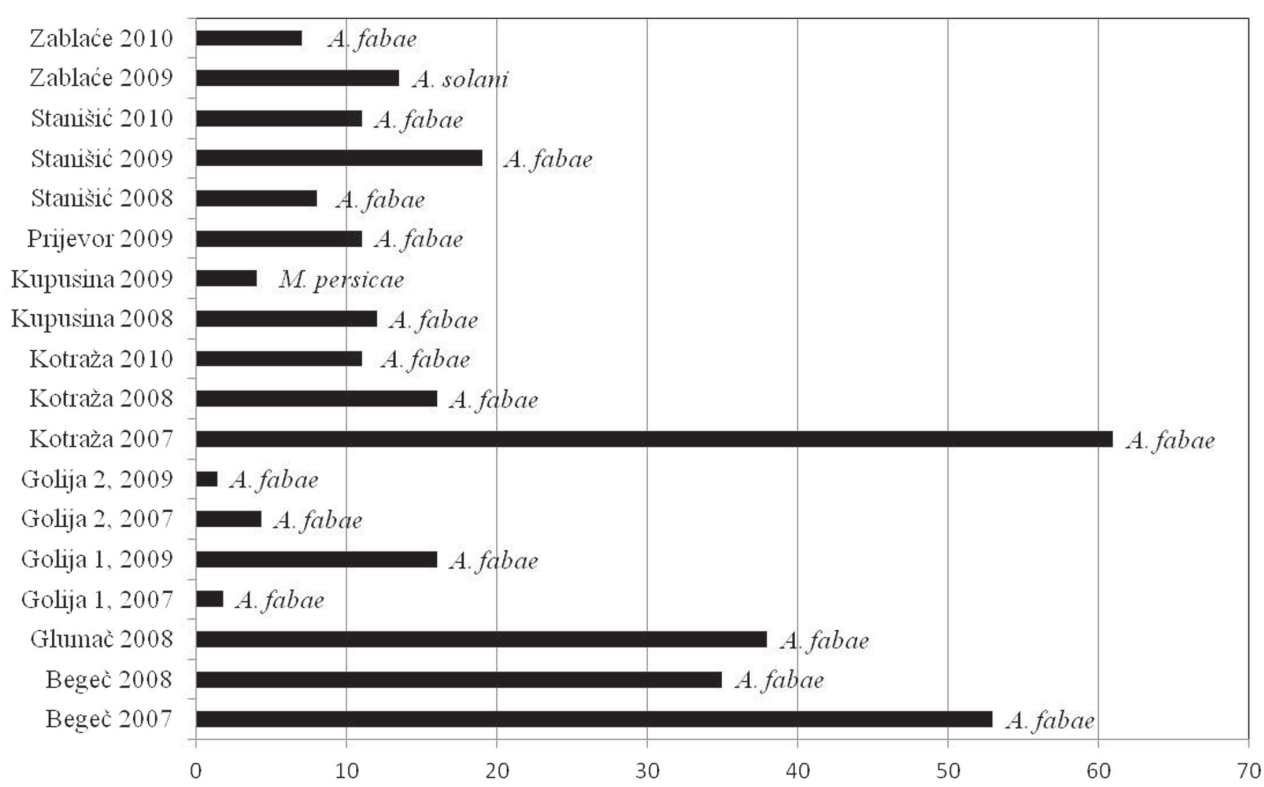

Figure 6. Vector pressure index for PLRV and most important vector per locality.

these high values were not repeated. Pressure of vectors for both viruses was constantly high in locality Begeč. In other localities, at the lowest altitudes, Kupusina and Stanišić, values of pressure vectors were low, but reached their maximums early in the season (Figs 5, 6).

\section{Discussion}

In the studies of biodiversity, in order to obtain a result with a scientific value, it is necessary to standardize sampling method, i.e. adapt it to target organisms. Different types of traps are the best known and the most widely used methods of sampling (Duelli 1997). Yellow water traps are often used for monitoring of aphid flight activities in potato fields (Sigvald 1989, Milošević and Petrović 1997, Kuroli and Lantos 2006, Kotzampigikis et al. 2008), but had not been used before for the studies of biodiversity of aphids. During these studies, they proved to be a good method, giving valid results. Shannon-Weaver diversity index is not an absolute indicator of the diversity of a certain area, it is used to compare different localities or the same locality in different years or weeks of a year. For this purpose it was used in these researches.

The values of Shannon-Weaver diversity index varied during the growing season. Temperature changes and rain influence the abundance of aphids (Morgan 2000), which also results in changes in the values of this index. However, in most localities the highest values were recorded in the first half of the monitored period, i.e. in spring. The total values of the index were similar among different sites, regardless of altitude. Due to the fact that Shannon-Weaver index takes into account 
the number of species and frequency of each species' individuals, localities with large differences in the number of aphids had similar index values. The highest values were recorded in locality Begeč, at an altitude of $80 \mathrm{~m}$. Morisita-Horn index takes into account the diversity of species and number of individuals, and it showed that there are not large differences between different localities, i.e. different localities have similar richness of the species. Locality Zablaće was clearly separated from other localities because of the five species which were recorded only in this locality during investigations.

In spite of similar values of Shannon-Weaver diversity index among different localities, participation of the vectors in it is different. The Chi-square analysis showed highly significant difference in vector frequencies among seasons and sites, with more pronounced differences for PVY. As a consequence of differences in vector frequencies, the vector pressure index in some regions was different also. In areas at lower altitudes such as Begeč, a higher number of vector species was registered, as well as more individuals of each present species. $M$. persicae, the most important vector of viruses was found in most localities, but in high number only in localities Begeč and Kotraža, while at the localities above $1100 \mathrm{~m}$ it was registered very rarely and in a low number. In localities under $900 \mathrm{~m}$, potato sowing is usually done in April, while there is an intensive growth of potato in May when aphid flight is at maximum and virus infection risk is the highest. Potato is the most sensitive in the first development phases, until flowering (DiFonso et al. 1994). Production of virus free seed potatoes is possible if the pressure of vectors does not exceed the value of $10-15$ by the end of June - early July (Basky 2002). In localities above $1000 \mathrm{~m}$, potato sowing is usually done at the end of May or beginning of Jun, depending on the ambient temperature. Except a lower number of aphids at higher altitudes, later sowing leads to avoiding the periods of aphid maximum flight and the risk of virus infection is reduced. Also, at higher altitudes, agricultural production is not intensive and the possibilities for isolated production are stronger.

Results of these studies show that only in localities at high altitudes, such as mountain Golija, it is possible to grow healthy, virus free seed potato. This research indicated that the potential of other mountainous regions of Serbia is also high and that Serbia has the capacity for production of quality seed potato. Also, this research may have relevance and application in other neighboring countries, too, because of similar relief, vegetation composition, composition of the fauna of aphids, and the possibility of crop production.

\section{Acknowledgements}

The research was supported by the Ministry of Education, Science and Technological Development of the Republic of Serbia (Project No. III 46008 - Development of integrated management of harmful organisms in plant production in order to overcome resistance and to improve food quality and safety). 


\section{References}

Basky Z (2002) The relationship between aphid dynamics and two prominent potato viruses (PVY and PLRV) in seed potatoes in Hungary. Crop Protection 21: 823-827. doi: 10.1016/S0261-2194(02)00045-5

DiFonso CD, Ragsdale EB, Radcliffe EB, Banttari EE (1994) Susceptibility to potato leafroll virus in potato: effects of cultivars, plant age at inoculation, and inoculation pressure on tuber infection. Plant Disease 78: 1173-1177. doi: 10.1094/PD-78-1173

Duelli P (1997) Biodiversity evaluation in agricultural landscapes: An approach at two different scales. Agriculture, Ecosystems and Environment 62: 81-91. doi: 10.1016/S01678809(96)01143-7

Jacky F, Bouchery Y (1988) Atlas des formes ailees des especes courantes de pucerons. INRA, 48 pp. Clarke KR (1993) Non-parametric multivariate analyses of changes in community structure. Australian Journal of Ecology 18: 117-143. doi: 10.1111/j.1442-9993.1993.tb00438.x

Kotzampigikis A, Hristova D, Tasheva-Terzieva E (2008) Distribution of Potato Leafroll Virus - (PLRV) and Potato Virus Y - (PVY) in a field experiment. Bulgarian Journal of Agricultural Science 14 (1): 56-67.

Krebs C (1989) Ecological Methodology. HarperCollins, New York, 654 pp.

Kuroli G, Lantos ZS (2006) Long-term Study of Alata Aphid Flight Activity and Abundance of Potato Colonizing Aphid Species. Acta Phytopatologica et Entomologica Hungarica 41 (3-4): 261-273. doi: 10.1556/APhyt.41.2006.3-4.9

Laznik Ž, Tóth T, Lakatos T, Vidrih M, Trdan S. (2010) Control of the Colorado potato beetle (Leptinotarsa decemlineata [Say]) on potato under field conditions: a comparison of the efficacy of foliar application of two strains of Steinernema feltiae (Filipjev) and spraying with thiametoxam. Journal of Plant Diseases and Protection 117 (3): 129-135

Magurran AE (2004) Measuring biological diversity. Oxford: Blackwell Science, 256 pp.

Morgan D (2000) Population dynamics of the bird cherry-oat aphid, Rhopalosiphum padi (L.), during the autumn and winter: a modelling approach. Agricultural and Forest Entomology (2): 297-304. doi: 10.1046/j.1461-9563.2000.00079.x

Milošević D, Petrović O (1997) A study of aphids flight activity (Homoptera, Aphididae) potential vectors of potato viruses. Acta Hort 462: 999-1006.

Petrović-Obradović O (2003) Biljne vaši (Homoptera: Aphididae) Srbije. Poljoprivredni fakultet Univerziteta u Beogradu, 153 pp. [in Serbian]

Petrović-Obradović O, Tomanović Ž, Poljaković-Pajnik L, Hrnčić S, Vučetić A, Radonjić S (2010) New invasive species of aphids (Hemiptera, Aphididae) in Serbia and Montenegro. Arch. Biol. Sci. Belgrade 62 (3): 775-780. doi: 10.2298/ABS1003775P

Robert I, Bourdin D (2001) Transmission of Viruses. Aphid transmission of Potato viruses. In: Loebenstein G, Berger PH, Brunt AA, Lawson RH: Virus and Virus - like Diseases of Potatoes and Production of Seed - Potatoes. Kluwer Academic Publishers, 195-226.

Remaudiere G, Seco Fernandez MV (1990) Claves para ayudar al reconocimiento de alados depulgones trampeados en la region mediterranea (Hom. Aphidoidea). Universidad De León, León, 2V, 205 pp. 
Salazar LF (1996) Potato Viruses and their Control. International Potato Center, Lima.

Sigvald R (1989) Relationship between aphid occurrence and spread of potato virus Y (PVY) in field experiments in southern Sweden. J. Appl. Ent. 108: 35-43.

Taylor LR (1984) A Handbook for Aphid Identification. (A Handbook for the Rapid Identification of the Alate Aphids of Great Britain and Europe). Roth. Exp. Stat., Harpenden, 171 pp. Van Harten A (1983) The relation between aphid flights and the spread of potato virus YN (PVYN) in the Netherlands. Potato Research 26: 1-15. http://aphmon.csl.gov.uk/info.cfm 